\title{
Preliminary outcomes of the ICIDOSE exercise and impact of the new models for occupational intakes.
}

\author{
Augusto Giussani ${ }^{1,}{ }^{*}$, Carlo-Maria Castellani ${ }^{2}$, Gareth Roberts $^{3}$, Andor Andrasi ${ }^{4}$, and Tamas \\ Pazmandi $^{4}$ \\ ${ }^{1}$ BfS - German Federal Office for Radiation Protection, 85764 Oberschleißheim, Germany \\ ${ }^{2}$ ENEA, Radiation Protection Institute, 40129 Bologna, Italy \\ ${ }^{3}$ Nuvia Ltd., Approved Dosimetry Services, Harwell OX11 ORL, United Kingdom \\ ${ }^{4}$ Hungarian Academy of Sciences Centre for Energy Research, Radiation Protection Department, \\ 1525 Budapest POB 49, Hungary
}

\section{ICIDOSE 2017}

In 2017 Working Group 7 of the European Radiation Dosimetry Group (EURADOS WG7) has organized an intercomparison action for internal dose assessment for occupational exposures. Main aim of the intercomparison action was to test the practical applicability of the "Technical Recommendations for Monitoring Individuals for Occupational Intakes of Radionuclides" ("TECHREC Recommendations") [1]

Among the four proposed cases, Case 1 (an artificial case of accidental inhalation of ${ }^{60} \mathrm{Co}$ ) was focussed on the impact of the changes introduced by the most recent ICRP recommendations [2], including the new report series on Occupational Intakes of Radionuclides (OIR) [3-5]. Additionally, the analysis of the results submitted for Case 4 (a complex real case of accidental inhalation of ${ }^{241} \mathrm{Am}$ ) provided useful feedbacks on the revised biokinetic models of ICRP.

\section{The new ICRP recommendations and their impact on incorporation monitoring}

In its 2007 recommendations ICRP introduced several changes affecting the estimation of the effective dose, among others new values for the radiation and for the tissue weighting factors, new definitions for the "remainder" tissue and for the calculation of the related equivalent dose, adoption of separate male and female reference computational phantoms and a new algorithm for the calculation of the effective dose. In addition to that, a new model for the Human Alimentary Tract (HAT), a revised model of the Human Respiratory Tract Model (HRTM) and updated systemic models for many radionuclides have been presented (3-6). All these changes affect not only the estimation of the effective dose, but also the calculation of the retention and excretion functions used to interpret the incorporation measurements.

* Corresponding author: agiussani@bfs.de 


\section{ICIDOSE Cases}

Case 1 was an artificial case purposely conceived to show that the two different approaches (analysis with current definitions and data vs. those adopted in the OIR reports) not only affect the final estimates of intake and dose, but also the procedure recommended by the TECHREC document for those estimates, requiring different levels of effort and expertise.

On the other side, the analysis of the results provided for Case 4 showed that the revised HRTM model and the OIR systemic model for americium radionuclides can successfully describe the retention data as measured in the real individuals. Following the procedure described in the IDEAS Guidelines (Version 2) for the Estimation of Committed Doses from Incorporation Monitoring Data [7], a very good fit of the data can be achieved with only slight changes of the HRTM americium specific parameters given in the draft of Part 4 of the OIR Report Series [8].

Finally, this analysis also enabled to check the applicability of the "dose per content" concept presented in the OIR Reports as an alternative method to estimate committed doses without the need to assess the related intake.

\section{References}

1. G. Etherington, A. Giussani, M.A. Lopez, P. Bérard, E. Blanchardon, B. Breustedt, C.M. Castellani, D. Franck, J. Marsh, D. Nosske, C. Challeton-de Vathaire. Technical Recommendations for Monitoring Individuals for Occupational Intakes of Radionuclides. EC Radiation Protection series (in press, 2018).

2. International Commission on Radiological Protection. The 2007 Recommendations of the International Commission on Radiological Protection. ICRP Publication 103. Ann. ICRP 37(2-4) (2007).

3. International Commission on Radiological Protection. Occupational Intakes of Radionuclides: Part 1. ICRP Publication 130. Ann. ICRP 44(2) (2015).

4. International Commission on Radiological Protection. Occupational Intakes of Radionuclides: Part 2. ICRP Publication 134. Ann. ICRP 45(3-4) (2016).

5. International Commission on Radiological Protection. Occupational Intakes of Radionuclides: Part 3. ICRP Publication 137. Ann. ICRP 46(3-4) (2017).

6. International Commission on Radiological Protection. Human Alimentray Tract Model for Radiation Protection. ICRP Publication 100. Ann. ICRP 36(1-2) (2006).

7. C.M. Castellani, J.W. Marsh, C. Hurtgen, E. Blanchardon, P. Berard, A. Giussani, M.A. Lopez, EURADOS report 2013-01 (2013) - available at http://www.eurados.org/en/Documents_Publications/Reports_documents

8. International Commission on Radiological Protection. Occupational Intakes of Radionuclides: Part 4. In preparation (Draft for public consultation published in July 2016). 